\title{
A DEFINITION OF AN UNKNOTTED SIMPLE CLOSED CURVE*
}

BY T. C. BENTON

Mazurkiewicz and Strasziewicz have given a definition of interlacability. $\uparrow$ That this definition can be altered to apply to a single curve so as to test for a knot in the curve will be shown in what follows.

Consider a three-dimensional, euclidean space. If for each point of the interval $t_{1} \leqq t \leqq t_{2}$ we let the continuous function $f(t)$ define a point in the space, the set of points so formed will be a continuous curve; If $f\left(t_{1}\right)=f\left(t_{2}\right)$ the curve is a closed curve. If $f\left(t^{\prime}\right)=f\left(t^{\prime \prime}\right)$ implies that either $t^{\prime}=t_{1}, t^{\prime \prime}=t_{2}$ or $t^{\prime}=t_{2}, t^{\prime \prime}=t_{1}$ or $t^{\prime}=t^{\prime \prime}$, then the curve is a simple closed curve and will be denoted by the symbol $\left[f(t) ; t_{1}, t_{2}\right]$.

Definition. If $\left[f(t) ; t_{1}, t_{2}\right]$ is a simple closed curve and if a uniformly continuous function $f(t, \lambda)$, where $t_{1} \leqq t \leqq t_{2}, 0 \leqq \lambda \leqq 1$, can be found having the following properties:

$$
\begin{aligned}
f(t, 1) & =f(t) \\
f(t, 0) & =f_{0}, \quad \text { a constant } \\
f\left(t^{\prime}, \lambda^{\prime}\right) & =f\left(t^{\prime \prime}, \lambda^{\prime \prime}\right),
\end{aligned}
$$

if and only if (i) $\lambda^{\prime}=\lambda^{\prime \prime}=0$, or (ii) $\lambda^{\prime}=\lambda^{\prime \prime} \neq 0$, and one of the following hold: (a) $t^{\prime}=t^{\prime \prime}$, or (b) $t^{\prime}=t_{1}, t^{\prime \prime}=t_{2}$, or (c) $t^{\prime}=t_{2}$, $t^{\prime \prime}=t_{1}$; then the simple closed curve $\left[f(t) ; t_{1}, t_{2}\right]$ is unknotted.

It must now be shown that the ordinary properties of knots are impossible for curves satisfying this definition; that is, no knotted curve can be a subset of a set which is in 1-1 continuous correspondence with a plane and every unknotted curve can be exhibited as a subset of such a set.

* Presented to the Society, March 30, 1929.

$\dagger$ Mazurkiewicz and Strasziewicz, Sur les coupures de l'espace, Fundamenta Mathematicae, vol. 9, p. 205. 
TheOREM 1. An unknotted simple closed curve can be exhibited as a subset of a set which is in 1-1 continuous correspondence with a plane.

Proof. Consider the set $[f(\bar{t}, \lambda)]$, where $\bar{t}$ is a constant and $\lambda$ varies. If $\bar{t}, t^{\prime}(\neq \bar{t})$ are not both members of the pair of values $t_{1}, t_{2}$, this set $[f(\bar{t}, \lambda)]$ has no point in common with $\left[f\left(t^{\prime}, \lambda\right)\right]$ except $f_{0}$. Also each of these sets is an arc from $f_{0}$ to a point of $\left[f(t) ; t_{1}, t_{2}\right]$ since their points are in 1-1 continuous correspondence with the points of the segment $0 \leqq \lambda \leqq 1$, and $f(\bar{t}, 0)$ $=f\left(t^{\prime}, 0\right)=f_{0}$.

From the fact that $f(t, \lambda)$ is uniformly continuous, it is necessary that the system of $\operatorname{arcs}[f(t, \lambda)], t_{1} \leqq t \leqq t_{2}$, be selfcompact. Moreover the arcs $\left[f\left(t_{1}, \lambda\right)\right]$ and $\left[f\left(t_{2}, \lambda\right)\right]$ are identical.

It will now be shown that the system of arcs is an equicontinuous system.* Suppose they do not form such a system. This means that there exists an $\epsilon$ such that no matter how small $\delta_{\epsilon 1}$ we choose, it must be possible to find an arc of the system which contains two points $f\left(t_{1}^{\prime}, \lambda_{11}\right), f\left(t_{1}^{\prime}, \lambda_{12}\right)$ such that these points are at a distance $<\delta_{\epsilon 1}$ apart, but there exists a value $\lambda_{13}$ where $\lambda_{11}<\lambda_{13}<\lambda_{12}$, and the distance from $f\left(t_{1}^{\prime}, \lambda_{13}\right)$ to $f\left(t_{1}^{\prime}, \lambda_{11}\right)$ is $>\epsilon$. For the arc $\left[f\left(t_{1}^{\prime}, \lambda\right)\right]$, however, there exists a $\delta_{1}$ such that for all values of $\delta<\delta_{1}$ every pair of points at distance $\leqq \delta$ apart can be joined by an arc of length $<\epsilon$, which joining arc is a subarc of the original. Hence if we choose $\delta_{\epsilon 2}$ less than the smaller of the two $\delta_{1}, \delta_{\epsilon 1}$, we can be sure that there is an arc $\left[f\left(t_{2}^{\prime}, \lambda\right)\right]$ distinct from $\left[f\left(t_{1}^{\prime}, \lambda\right)\right]$ for which there are two points $f\left(t_{2}^{\prime}, \lambda_{21}\right), f\left(t_{2}^{\prime}, \lambda_{22}\right)$ at a distance $<\delta_{\epsilon 2}$ apart but on which there also exists a point $f\left(t_{2}^{\prime}, \lambda_{23}\right)$ such that $\lambda_{21}<\lambda_{23}<\lambda_{22}$ and the distance from $f\left(t_{2}^{\prime}, \lambda_{23}\right)$ to $f\left(t_{2}^{\prime}, \lambda_{21}\right)$ is greater than $\epsilon$. By a proper choice of $\delta_{\epsilon 3}$ we proceed with this process and since the set of arcs was supposed not equicontinuous we must arrive at an infinite set of arcs. Since all values of $t$ lie between $t_{1}$ and $t_{2}$, the sequence $t_{1}^{\prime}, t_{2}^{\prime}, t_{3}^{\prime}, \cdots$ must have a limit $T$. As we can discard members of this sequence until $T$ is the only limit, it will be supposed that this has been done and that $T$ is the sequential limit of the sequence $t_{1}^{\prime}, \ldots$. In the same way it may be supposed that the sequence $\lambda_{1 i}, \lambda_{2 i}, \lambda_{3 i}, \cdots$ must have

* For definition see R. L. Moore, On the generation of a surface, Fundamenta Mathematicae, vol. 4, p. 106, footnote 3. 
a unique limit $\Lambda_{i}$ for $i=1,2,3$. Now on the $\operatorname{arc}[f(T, \lambda)]$ we can make the point $f\left(T, \Lambda_{1}\right)$ the same as the point $f\left(T, \Lambda_{2}\right)$ because the sequence $\delta_{\epsilon 1}, \delta_{\epsilon 2}, \delta_{\epsilon 3}, \cdots$ can be chosen to converge to zero and still satisfy all other conditions so far imposed on it, and then $\left|\lambda_{j 1}-\lambda_{j 2}\right| \rightarrow 0$ as $j \rightarrow \infty$. But since we choose $\lambda_{j 1}<\lambda_{j 3}<\lambda_{j 2}$ we must also have $\Lambda_{3}=\Lambda_{1}$. This, however, contradicts the fact that the distance from $f\left(t_{j}^{\prime}, \lambda_{j 1}\right)$ to $f\left(t_{j}^{\prime}, \lambda_{j 2}\right)$ was chosen greater than $\epsilon$ for every $j$, because this choice means that the smallest possible distance from $f\left(T, \Lambda_{1}\right)$ to $f\left(T, \Lambda_{3}\right)$ is $\epsilon$. This shows that the assumption that the arcs do not form an equicontinuous system is false.

By a theorem due to R. L. Moore* this set of $\operatorname{arcs}[f(t, \lambda)]$ is in 1-1 continuous correspondence with a plane set composed of a circle plus its interior. From this the theorem follows.

Theorem 2. Any simple closed curve $M$ in a set $S$, which set $S$ is in 1-1 continuous correspondence with a plane set $S^{\prime}$ consisting of a circle plus its interior, must be unknotted.

Proof. Let $S^{\prime}$ be a circle $C$ of radius one about the point $O$, together with its interior. Let $M^{\prime}$ denote the set of points in $S^{\prime}$ that correspond to $M$ under the given correspondence. Let the set of points of $S$ that correspond to the interior of $M^{\prime}$ in the plane be called the interior of $M$. By a theorem due to Schönflies it is possible to make $M$ plus its interior correspond to $C$ plus its interior. $\dagger$ Then the straight line segment from $O$ to a point of $C$ will correspond to an arc from $f_{0}$ (which can be made the correspondent of $O$ ) to that point of $M$ which is the correspondent of the point of $C$ on the straight line segment. These segments enable us to set up a polar coordinate system on the set $S^{\prime}$. If $f$ is the function which gives the correspondence between $S$ and $S^{\prime}$, we see that if a point $(\theta, r)$ is given on $S^{\prime}$, $f(\theta, r)$ will be its correspondent on $S$. Since $f(0, r)=f(2 \pi, r)$ and the other properties of the function used in the definition of an unknotted curve are satisfied if $0 \leqq \theta \leqq 2 \pi$ and $0 \leqq r \leqq 1, M$ must be unknotted.

YALE UNIVERSITY

* R. L. Moore, On the generation of a surface, loc. cit., p. 117, Theorem 3.

† See J. R. Kline, Proceedings of the National Academy of Sciences, vol. 6 (1920), p. 530, for a statement of this theorem. 